\title{
Knockdown of COBRA1 decreases the proliferation and migration of hepatocellular carcinoma cells
}

\author{
EMAN EL ZENEINI ${ }^{1}$, SARAH KAMEL ${ }^{1}$, MAHMOUD EL-METEINI ${ }^{2}$ and ASMA AMLEH ${ }^{1,3}$ \\ ${ }^{1}$ Biotechnology Department, The American University in Cairo, New Cairo 11835; \\ ${ }^{2}$ HPB and Liver Transplant Surgical Department, Faculty of Medicine, Ain Shams University, \\ Cairo 11341; ${ }^{3}$ Department of Biology, School of Sciences and Engineering, \\ The American University in Cairo, New Cairo 11835, Egypt
}

Received August 10, 2016; Accepted December 27, 2016

DOI: $10.3892 /$ or.2017.5390

\begin{abstract}
Cofactor of BRCA1 (COBRA1) is one of the four subunits that make up the negative elongation factor (NELF) complex that is involved in the stalling of RNA polymerase II early during transcription elongation. As such, it regulates the expression of a substantial number of genes involved in cell cycle control, cellular metabolism and DNA repair. With no DNA binding domain, its capacity to modulate gene expression occurs via its ability to interact with different transcription factors. In the field of cancer, its role is not yet fully understood. In this study, we demonstrate the frequent overexpression of COBRA1 along with the remaining NELF subunits in hepatocellular carcinoma (HCC) tissues relative to non-cancerous liver tissues. To elucidate its biological significance in HCC, RNA interference was utilized to silence COBRA1 expression in the HCC cell line, HepG2. Interestingly, COBRA1 knockdown resulted in a significant decrease in cell proliferation and migration, accompanied by a concomitant reduction in the expression of the proliferation marker, Ki-67. Survivin, a proto-oncogene that is commonly upregulated in almost all human malignancies including HCC, was also significantly downregulated following COBRA1 silencing. This suggests that it might be one of the mechanisms by which COBRA1 mediates its role in HCC. Taken together, our data findings collectively highlight an important role for COBRA1 in supporting HCC proliferation and migration.
\end{abstract}

Correspondence to: Dr Asma Amleh, Department of Biology, School of Sciences and Engineering, The American University in Cairo, AUC Avenue, P.O. Box 74, New Cairo 11835, Egypt

E-mail: aamleh@aucegypt.edu

Abbreviations: AR, androgen receptor; COBRA1, cofactor of BRCA1; ER $\alpha$, estrogen receptor- $\alpha$; GR, glucocorticoid receptor; HCC, hepatocellular carcinoma; IAP, inhibitor of apoptosis; NELF, negative elongation factor; PRB, progesterone receptor $\mathrm{B}$; RDBP, RD RNA binding protein; WT, wild-type

Key words: NELF-B, negative elongation factor complex, negative elongation factor, hepatocellular carcinoma

\section{Introduction}

Liver cancer is the second most frequent cause of cancer-related deaths worldwide, responsible for more than 0.5 million deaths globally and 0.5-1 million newly diagnosed cases each year $(1,2)$. Hepatocellular carcinoma (HCC) accounts for $70-85 \%$ of all presented liver cancer cases, and is considered one of the most aggressive cancers worldwide (1). The lethal nature of HCC stems from the aggressive course of the disease combined with the late diagnosis and the high risk of tumor recurrence $(3,4)$. In addition, due to the heterogeneity and underlying complexity of HCC, the molecular mechanisms underlying its development and progression remain largely unknown (5).

Cofactor of BRCA1 or COBRA1 was originally identified as a novel BRCA1 interacting protein through a yeast two-hybrid screen (6). It was not until later that COBRA1 was identified as the same protein as NELF-B, one of the four subunits that make up the human negative elongation factor (NELF) complex. Upon the assembly of the four subunits NELF-A, NELF-B (COBRA1), NELF-C/D and NELF-E, the NELF complex is activated and recruited together with the DRB sensitivity-inducing factor (DSIF) to induce the stalling of RNAPII early during transcription elongation. It is believed that NELF recruitment occurs immediately after transcription initiation and acts to stall the elongation of the RNA transcript in a process referred to as promoter-proximal pausing (7). As such, it is responsible for the regulation of a significant number of genes involved in cell cycle control, proliferation, metabolism as well as cellular responses to stimuli and stress (8).

In eukaryotes, COBRA1's role in the regulation of gene expression is not limited by any means to its interaction with BRCA1 and the remaining NELF subunits. With no DNA binding domain of its own, its ability to regulate genes occurs via its interaction with other transcription factors that bind to specific DNA regulatory sequences. In fact, Aiyar et al demonstrated a novel role for COBRA1 as a transcriptional corepressor in the repression of hormone-responsive transcription (9). In response to estrogen, COBRA1 binds directly to the activated estrogen receptor- $\alpha(\mathrm{ER} \alpha)$ and is recruited as NELF-B along with the rest of the NELF complex to the promoters of a subset of estrogen-responsive genes where it acts to stall RNAPII and repress ER $\alpha$-mediated transcription (9). Given the positive 
role ER $\alpha$-mediated signaling has on the development of breast cancer $(10,11)$, COBRA1's physiological role in suppressing the estrogen-dependent growth of breast cancer cells was illustrated (9). In addition to ER $\alpha$, COBRA1 binds with various degrees of affinity to other nuclear receptors as well such as the androgen receptor (AR), progesterone receptor $\mathrm{B}(\mathrm{PRB})$ and the glucocorticoid receptor (GR). Among these, the AR was shown to exhibit the strongest affinity for COBRA1 and thus, the most susceptible to the COBRA1-mediated repression of AR transcription (12). Similar to the ER $\alpha$ pathway, AR-mediated signaling is well established in mediating the development and/or progression of different malignancies such as prostate cancer and HCC $(13,14)$. However, the role of COBRA1 in these malignancies has not been yet studied and remains unknown.

As an integral subunit of the major transcriptional complex NELF and the ability to modulate nuclear receptormediated transcription, COBRA1 can regulate a substantial number of genes involved in different pathways $(15,16)$. Even though it has been extensively studied over the years, in the field of cancer it is not yet fully understood. To date, it has only been examined in breast and upper gastrointestinal cancer. In breast cancer, several lines of evidence highlight COBRA1 as a tumor suppressor (17). In contrast, a significant overexpression of COBRA1 was reported in $79 \%$ of upper gastrointestinal cancers (UGCs) implicating COBRA1 as a novel oncogene in UGC (18). In view of COBRA1's role in the regulation of androgen-dependent transcription, we examined the role of COBRA1 in HCC. Here, we provide lines of evidence for the first time to highlight an important role for COBRA1 in supporting HCC proliferation and migration. Our data findings suggest a potential link between COBRA1 and the inhibitor of apoptosis (IAP) family member, survivin, that is commonly upregulated in almost all human malignancies including HCC.

\section{Materials and methods}

Tissue samples. Fifteen hepatocellular carcinoma tissue samples and their paired adjacent non-neoplastic liver samples were collected from patients undergoing resection or transplantation surgery at Ain Shams Specialized Hospital, Cairo, Egypt. Written informed consents were obtained from all patients. Ethics approval was from the Institutional Review Board (IRB) of the American University in Cairo. Characteristics of the HCC samples collected from the patient cohort included in this study are summarized in Table I. The majority of the HCC samples collected were from an HCV background, with one sample in particular, having a mixed $\mathrm{HBV}$ and $\mathrm{HCV}$ etiology. Five patients received preoperative treatment in the form of either radiofrequency ablation, transarterial chemoembolization and/or microwave ablation while the remaining did not. All collected tissue specimens were frozen immediately on dry ice and stored at $-80^{\circ} \mathrm{C}$ for further RNA and protein analysis.

Cell lines and culture. The human HCC cell line, HepG2, was kindly provided by Dr Mehmet Ozturk from the Department of Molecular Biology and Genetics, Bilkent University, Turkey. Cells were cultured in complete medium composed of RPMI-1640 (Invitrogen, USA) supplemented with 10\% fetal
Table I. Characteristics of patient cohort.

\begin{tabular}{lc}
\hline Characteristics & No. of patients \\
\hline Total & 15 \\
Age (years) & $45-65$ \\
Range & \\
Gender & 12 \\
Male & 3 \\
Female & \\
Preoperative treatment & 5 \\
Yes & 10 \\
No & \\
Etiology & 1 \\
HBV and HCV & 10 \\
HCV & \\
Grade & \\
I & 0 \\
II & 14 \\
III/IV & 0
\end{tabular}

Child Pugh class

$\begin{array}{ll}\text { A } & 8 \\ \text { B } & 4 \\ \text { C } & 3\end{array}$

$\mathrm{HBV}$, hepatitis B virus; $\mathrm{HCV}$, hepatitis $\mathrm{C}$ virus.

Table II. Target sequences and exons of COBRA1 siRNAs used in the study.

\begin{tabular}{lcc}
\hline $\begin{array}{l}\text { siRNA } \\
\text { identifier }\end{array}$ & Target sequence (5'-3') & $\begin{array}{c}\text { Target } \\
\text { exon }\end{array}$ \\
\hline siGENOME & 1 CCGAAAGCUUCACUAAGUU & 9 and 10 \\
COBRA1 & 2 GCGACUUGGCCUUUGGCGA & 11 \\
SMARTPool & 3 GAGCCUGGGACAUGAUCGA & 8 \\
(M-015839-00) & 4 CGUCUAAGCUGGAGGCGUU & 12 \\
\hline
\end{tabular}

bovine serum (Invitrogen), $100 \mathrm{U} / \mathrm{ml}$ penicillin and $100 \mathrm{mg} / \mathrm{ml}$ streptomycin (Invitrogen), and incubated in a humidified incubator at $37^{\circ} \mathrm{C}$ with $5 \% \mathrm{CO}_{2}$.

Small interference RNA (siRNA). COBRA1 siGENOME SMARTPool siRNA (M-015839-00; Table II) was purchased from Dharmacon and AllStars negative control siRNA (SI03650318) was purchased from Qiagen. All siRNAs were resuspended in RNase-free water to a final concentration of $20 \mu \mathrm{M}$. Approximately $2.5 \times 10^{5}$ cells were reverse transfected with $25 \mathrm{nM}$ siRNA using $5 \mu$ l of Lipofectamine RNAiMAX (Life Technologies, USA) in a 6-well plate according to the manufacturer's protocol. Growth media were replenished $24 \mathrm{~h}$ 
Table III. Primer sequences, amplicon sizes and PCR conditions (annealing temperature, number of cycles).

\begin{tabular}{|c|c|c|c|}
\hline Gene name & Primer sequence $\left(5^{\prime}-3^{\prime}\right)$ & Amplicon size (bp) & PCR conditions \\
\hline \multirow[t]{2}{*}{ B-ACTIN } & F: GCAAAGACCTGTACGCCAAC & 777 & $58^{\circ} \mathrm{C}, 27$ cycles \\
\hline & R: GAGACCAAAAGCCTTCATACATCTC & & \\
\hline \multirow[t]{2}{*}{ COBRA1 } & F: ACATCACCAAGCAGAGGAA & 366 & $59.5^{\circ} \mathrm{C}, 32$ cycles \\
\hline & R: GATCCAGCTGTTCCAGCTTC & & \\
\hline \multirow[t]{2}{*}{ NELF-A } & F: GTCGGCAGTGAAGCTCAAGT & 250 & $60^{\circ} \mathrm{C}, 35$ cycles \\
\hline & R: TTCACACTCACCCACCTTTTCT & & \\
\hline \multirow[t]{2}{*}{ NELF-C/D } & F: GAAGAAGGAGAGACCCCAGC & 443 & $56^{\circ} \mathrm{C}, 28$ cycles \\
\hline & R: GTGCCCAAGGCTAGTGTGAT & & \\
\hline \multirow[t]{2}{*}{ NELF-E (19) } & F: TGGTGAAGTCAGGAGCCATCAG & 565 & $63^{\circ} \mathrm{C}, 28$ cycles \\
\hline & R: CGCCGTTCAGGGAATGAATC & & \\
\hline \multirow[t]{2}{*}{ Ki-67 (20) } & F: CTTTGGGTGCGACTTGACG & 199 & $60^{\circ} \mathrm{C}, 28$ cycles \\
\hline & R: GTCGACCCCGCTCCTTTT & & \\
\hline \multirow[t]{3}{*}{ BIRC5 (21) } & F: TTGAATCGCGGGACCCGTTGG & Isoform 1: 477 & $61^{\circ} \mathrm{C}, 27$ cycles \\
\hline & R: CAGAGGCCTCAATCCATGGCA & Isoform 2: 359 & \\
\hline & & Isoform 3: 546 & \\
\hline
\end{tabular}

F, forward primer; $\mathrm{R}$, reverse primer; bp, base pair.

after transfection. As controls, cells were either left untreated (blank) or treated with Lipofectamine only (mock). Cells were cultured for a total of $72 \mathrm{~h}$ and then harvested for total RNA and protein analysis.

Reverse transcription-polymerase chain reaction ( $R T-P C R)$. Total RNA was extracted using TRIzol reagent (Invitrogen). One microgram of total RNA was reverse transcribed using RevertAid First Strand cDNA Synthesis kit (Thermo Fisher Scientific, USA) and random primers in a final volume of $20 \mu \mathrm{l}$, according to the manufacturer's protocol. Analysis of differential gene expression was performed using semi-quantitative RT-PCR in a final volume of $25 \mu \mathrm{l}$, using $1 \mu \mathrm{l}$ template, $2.5 \mu 1$ 10X DreamTaq buffer, $0.5 \mu 1$ dNTPs $10 \mathrm{mM}$ (Thermo Fisher Scientific), $0.25 \mu 1$ DreamTaq DNA polymerase and $0.75 \mu \mathrm{l}$ of each primer $(10 \mu \mathrm{M})$. PCR reaction conditions were as follows; $95^{\circ} \mathrm{C}$ for $5 \mathrm{~min}$, followed by cycles of $95^{\circ} \mathrm{C}$ for $30 \mathrm{sec}$, annealing for $30 \mathrm{sec}$ and $72^{\circ} \mathrm{C}$ for $45 \mathrm{sec}$, and a final extension at $72^{\circ} \mathrm{C}$ for $7 \mathrm{~min}$. Primer sequences and PCR conditions are listed in Table III. For each set of primers, the optimal number of cycles was determined to ensure that the analysis was carried out within the exponential range of amplification. Amplified PCR products were run on $1.5-2 \%$ agarose gel and visualized using Gel Doc EZ System (BioRad, USA).

Immunoblotting. Cells were lysed with ice-cold Laemmli lysis buffer (50 mM Tris pH 6.8, 2\% SDS, $10 \%$ glycerol), supplemented with 1X Halt Protease Inhibitor Cocktail (Thermo Fisher Scientific). Protein concentrations were quantified using the BCA assay kit (Pierce Biotechnology, USA) according to the manufacturer's protocol. Equal amounts of whole cell lysates (20-50 $\mu \mathrm{g})$ were resolved using a $12 \%$ SDS-polyacrylamide gel and transferred onto nitrocellulose membranes. After blocking with 5\% non-fat dry milk, the membrane was incubated with rabbit polyclonal anti-COBRA1 (Abcam, ab48336; used for HCC tissue analysis) (2 $\mu \mathrm{g} / \mathrm{ml}$ in $3 \%$ non-fat dry milk in $0.01 \%$ PBST), rabbit monoclonal anti-COBRA1 (Abcam, ab167401; used for knockdown analysis) (1:1,000 in 5\% non-fat dry milk in $0.01 \%$ PBST), rabbit polyclonal anti-B-ACTIN (Pierce, PA1-46296) or mouse monoclonal anti-B-TUBULIN (Sigma, T7816) (1:20,000 in 5\% non-fat dry milk in $0.01 \%$ PBST) at $4{ }^{\circ} \mathrm{C}$ overnight. The membrane was then incubated with alkaline phosphatase-conjugated secondary antibodies and visualized using PhosphoGLO Substrate (KPL, USA).

Cell proliferation assay. Approximately $2.5 \times 10^{5}$ cells were reverse transfected with either COBRA1 siRNA or negative siRNA in a 6-well plate as earlier described. To monitor the rates of cellular proliferation, the cells were harvested at 24-h intervals for four days and the cell count determined using the trypan blue exclusion assay. Briefly, an aliquot of the cell suspension was mixed with an equal volume of $0.4 \%$ trypan blue in phosphate-buffered saline and the number of cells determined using a hemocytometer (Hausser Scientific, USA).

Scratch wound healing assay. Differences in cell migration were assessed via a classical scratch wound healing assay, which is widely used to evaluate cell migration as previously described (22). Twenty-four hours post-transfection, cells were reseeded in 24-well plates. At $72 \mathrm{~h}$ post-transfection, the cell monolayer was carefully scraped using a $20-\mu 1$ pipette tip. Cells were washed with PBS and incubated for another $24 \mathrm{~h}$. Migration of cells was monitored with an inverted microscope. 
Table IV. Oncomine microarray datasets used in this study.

\begin{tabular}{|c|c|c|c|c|c|}
\hline Study & Sample type & Patients (n) & Patients/sample & Year of study & Refs. \\
\hline \multirow[t]{4}{*}{ Chen et al, liver } & Normal liver & 187 & 76 & 2002 & (25) \\
\hline & Focal nodular hyperplasia & & 4 & & \\
\hline & Hepatocellular adenoma & & 3 & & \\
\hline & Hepatocellular carcinoma & & 104 & & \\
\hline \multirow[t]{3}{*}{ Mas et al, liver } & Normal liver & 115 & 19 & 2009 & (26) \\
\hline & Cirrhotic & & 58 & & \\
\hline & Hepatocellular carcinoma & & 38 & & \\
\hline \multirow[t]{2}{*}{ Roessler et al, liver } & Paired non-tumor & 43 & 21 & 2010 & (27) \\
\hline & Hepatocellular carcinoma & & 22 & & \\
\hline \multirow[t]{2}{*}{ Roessler et al, liver 2} & Paired non-tumor & 445 & 220 & 2010 & (27) \\
\hline & Hepatocellular carcinoma & & 225 & & \\
\hline \multirow[t]{4}{*}{ Wurmbach et al, liver } & Normal liver & 75 & 10 & 2007 & (28) \\
\hline & Cirrhotic & & 13 & & \\
\hline & Liver cell dysplasia & & 17 & & \\
\hline & Hepatocellular carcinoma & & 35 & & \\
\hline
\end{tabular}

The wound area was analyzed using TScratch software (23), and the percentage wound closure rate was calculated as follows: Percentage wound closure $=[$ (wound area at $0 \mathrm{~h}$ wound area at $24 \mathrm{~h}$ ) / wound area at $0 \mathrm{~h}] \times 100 \%$.

Data mining. The Oncomine ${ }^{\mathrm{TM}}$ cancer microarray database (http://www.oncomine.org/) (24) was used to query the expression profile of the different NELF subunits in HCC tissues versus their normal counterparts. HCC microarray datasets utilized in this study are summarized in Table IV (25-28). Statistical significance was automatically computed by the default Oncomine algorithms using a two-tailed Student's t-test. Only analyses that illustrated a fold change of $\geq 1.4$ and $\mathrm{p}<0.05$ was included. The Genevestigator meta-analysis database (https://genevestigator.com/) (29) was utilized to mine the differential expression of COBRA1 across different HCC cell lines.

Data analysis. Densitometric analysis was performed using ImageJ Software (National Institute of Health, USA, http:// www.imagej.nih.gov/ij). For both the PCR and western blotting, quantified band intensities were normalized to the loading control B-ACTIN or B-TUBULIN, respectively. Relative changes in gene expression are expressed as fold change from the negative siRNA-transfected cells (control), unless specified otherwise. All statistical analyses were performed using GraphPad Prism 5.0 (GraphPad Software Inc, San Diego, CA, http://www.graphpad.com/). All values represent the mean \pm standard deviation (SD) from three independent experiments unless specified otherwise. For pairwise comparisons, statistical significance was assessed using the Student's t-test (two-tailed). One-way or a two-way ANOVA, followed by Bonferroni post-test was used to analyze differences between multiple experimental groups. A p-value $<0.05$ was considered significant $\left({ }^{*} \mathrm{p}<0.05 ;{ }^{* *} \mathrm{p}<0.01 ;{ }^{* * *} \mathrm{p}<0.001 ;{ }^{* * * *} \mathrm{p}<0.0001\right)$.

\section{Results}

COBRA1 expression is frequently upregulated in human hepatocellular carcinoma. Using RT-PCR, we assessed the expression of COBRA1 mRNA in 15 paired HCC tissue samples and their adjacent non-tumor tissues. Upregulated COBRA1 expression ( $\geq 1.5$-fold) was observed in $66.6 \%$ $(10 / 15)$ of HCC samples with a statistically significant mean increase of 1.5-fold (paired t-test, $\mathrm{p}<0.05$, Fig. 1A and B). Analysis of protein expression in the same set also revealed upregulation of COBRA1 protein expression in 53\% (8/15) of HCC samples (Fig. 1C and D). However, given the small sample size, the mean increase in expression at the protein level was not statistically significant $(\mathrm{p}>0.05)$. These results suggested the frequent overexpression of COBRA1 in HCC tissues. So, to further explore this on a larger scale, we performed in silico analysis of HCC microarray datasets in the Oncomine database. A query search on the differential expression of COBRA1 in HCC tumor tissues versus normal liver tissue yielded results from four different datasets (26-28). Although the study of Mas et al (26) revealed no significant difference in COBRA1 expression, the analysis of the three remaining datasets showed a significant overexpression of COBRA1 mRNA in HCC tumor tissues versus their normal counterparts ( $\mathrm{p} \leq 0.05)$ (Fig. 2). Both datasets from the study of Roessler et al (27) show comparable results, with a fold change of $1.614(\mathrm{p}=2.61 \mathrm{E}-43)$ and $1.635(\mathrm{p}=5.29 \mathrm{E}-6)$. Similarly, in the study of Wurmbach et al (28), even though no significant difference exists between normal tissue and either cirrhotic or dysplastic tissue, overexpression of COBRA1 mRNA is observed in HCC tumor tissues versus normal liver tissue with a 1.402 -fold change $(p=0.002)$. To test whether the observed overexpression could be due to COBRA1 gene amplification, we utilized the database again to analyze the copy numbers of COBRA1 in HCC tumors versus normal liver tissue. Data 
A

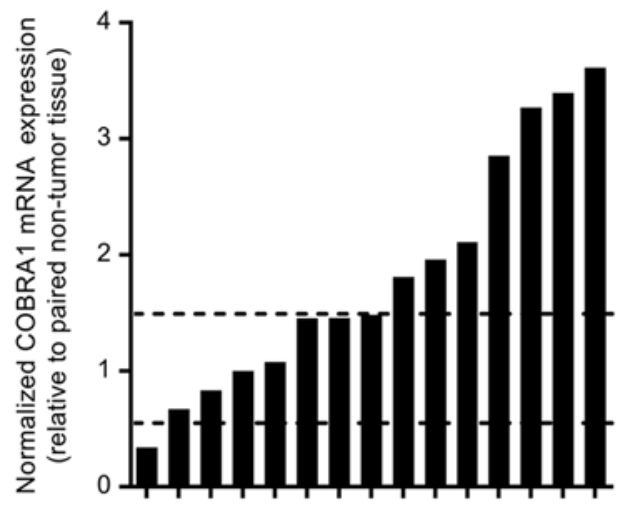

HCC samples $(n=15)$

C

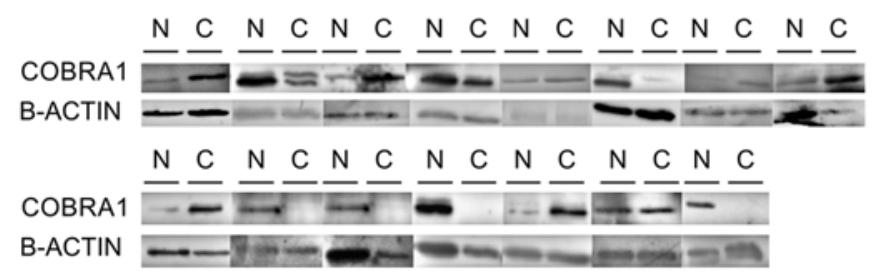

B

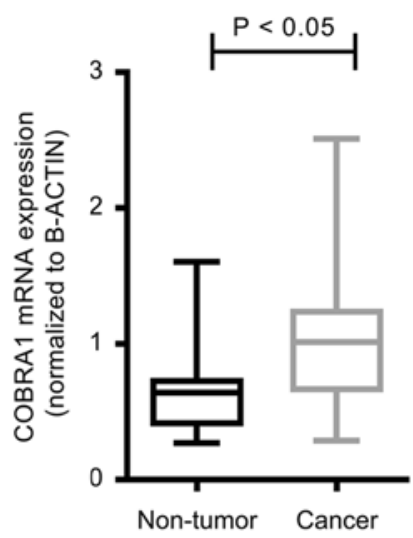

D

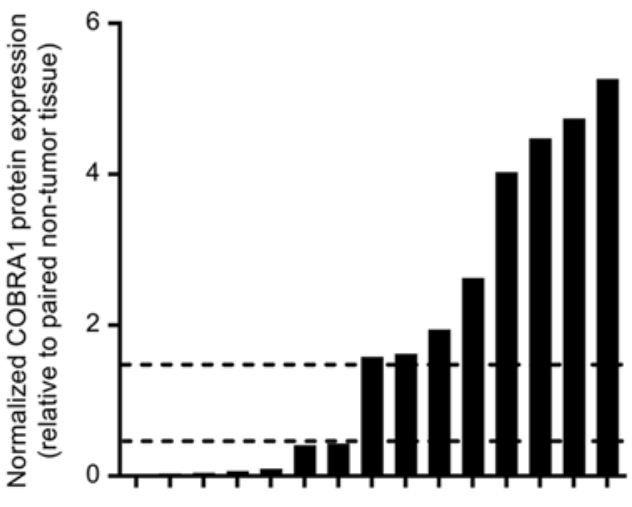

HCC samples $(n=15)$

Figure 1. Expression of COBRA1 (NELF-B) mRNA and protein in human hepatocellular carcinoma tissue samples. (A) Normalized COBRA1 mRNA expression in tumor tissues is expressed as fold change relative to paired non-tumor tissues (tumor COBRA1/normal COBRA1). A fold change of $\leq 0.5$ indicates a downregulation in COBRA1 expression while a fold change $\geq 1.5$-fold change indicates an upregulation in COBRA1 expression. (B) A paired Student's t-test was used to analyze the difference in COBRA1 mRNA expression in tumor tissues vs paired non-tumor tissues. Statistically significant at ${ }^{*} \mathrm{p}<0.05$. (C) Western blotting gels showing the expression of COBRA1 protein in tumor tissues (C) vs their paired non-tumor tissues (N). (D) Normalized COBRA1 protein expression in tumor tissues is expressed as fold change relative to paired non-tumor tissues.

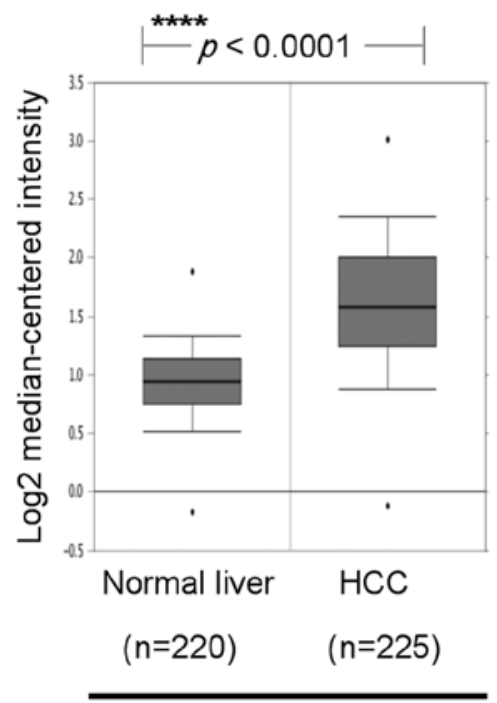

Roessler liver 2

Fold change: 1.614



Roessler liver

Fold change: 1.635



Wurmbach liver

Fold change: 1.402

Figure 2. Overexpression of COBRA1 (NELF-B) mRNA in hepatocellular carcinoma versus normal liver tissue. The differential expression of COBRA1 mRNA in hepatocellular carcinoma tissue samples versus normal liver tissue as per the Oncomine cancer database (http://www.oncomine.org (24). Results are illustrated as boxplots, with the box representing the lower quartile, median and upper quartile, respectively and the bars representing the 10th and 90th percentile. The dots represent the minimum and maximum values. For each study, the number of patients in each category is indicated in brackets as well as the fold change and $\mathrm{p}$-value as determined by the Student's t-test. ${ }^{*} \mathrm{p}<0.05 ;{ }^{* * * *} \mathrm{p}<0.0001$. 


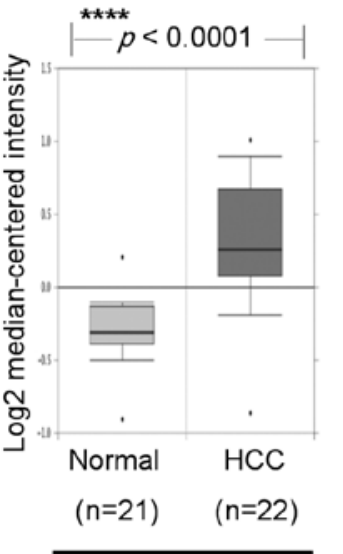

Roessler liver

Fold change: 1.514

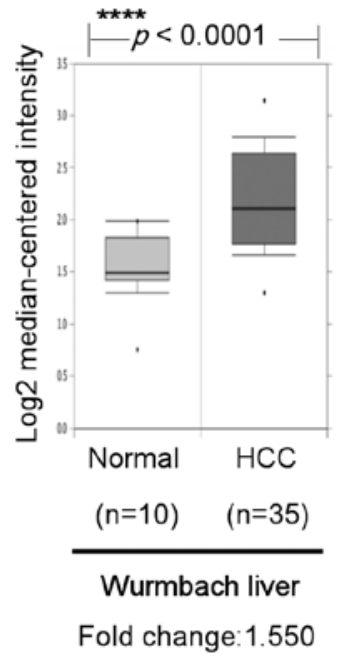

NELF-A

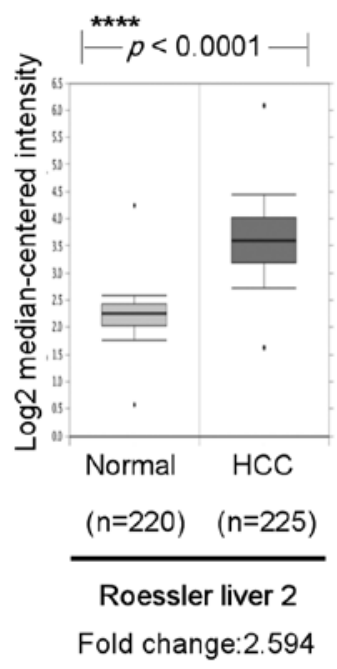

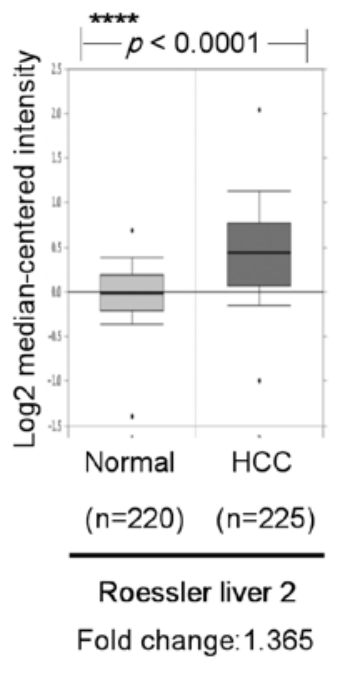

Fold change: 1.365



NELF-C/D

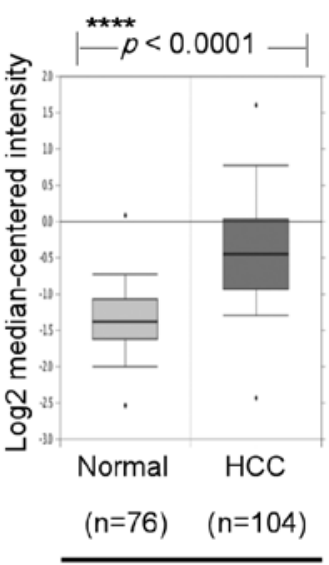

Chen liver

Fold change: 1.896

\section{NELF-E}

Figure 3. Overexpression of the different NELF subunits in hepatocellular carcinoma versus normal liver tissue. The differential expression of NELF-A, NELF-C/D and NELF-E was assessed in HCC tissue samples versus normal liver tissue in the same datasets queried previously for COBRA1 in the Oncomine database (http://www.oncomine.org/) (24). Data for NELF-C/D was only available from the Wurmbach et al dataset (28). The Chen et al study showed data only for the NELF-E subunit (25). For each study, the number of patients in each category is indicated in brackets as well as the fold change and p-value as determined by the Student's t-test. ${ }^{* * * * *} \mathrm{p}<0.0001$.

from three datasets revealed no significant difference in the copy numbers of COBRA1 between normal tissue and HCC tumors (data not shown).

Expression of NELF subunits is upregulated in hepatocellular carcinoma. The COBRA1 or NELF-B is an essential structural and functional component of the NELF complex (7). Given the previously established interdependent nature of the NELF subunits $(8,17,30)$, we were intrigued whether the overexpression of COBRA1 in HCC would also be accompanied by a similar deregulation in the expression of the remaining NELF subunits, NELF-A, NELF-C/D and NELF-E. Utilizing the Oncomine database to query the same datasets previously queried for
COBRA1, a similar pattern of overexpression was observed for all NELF subunits (Fig. 3). The datasets that showed a significant overexpression of COBRA1 revealed a similar pattern of overexpression for the remaining subunits as well. While there was data from only one dataset to support an overexpression pattern for the NELF-C/D subunit, the overexpression of the NELF-E subunit was backed up by four different datasets. It is important to mention that out of the different NELF subunits, the NELF-E subunit demonstrated the highest differential expression pattern in HCC tissues versus normal liver tissue with a-fold change ranging from 1.8 to 2.7. Similar to COBRA1, there was no significant difference in the expression of the different NELF subunits between normal liver tissue and either 
A
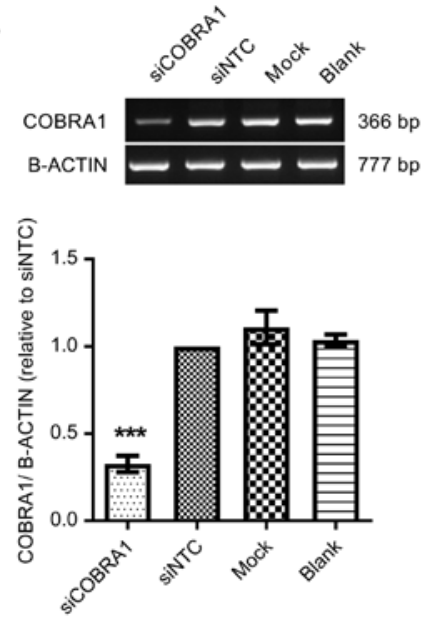

B
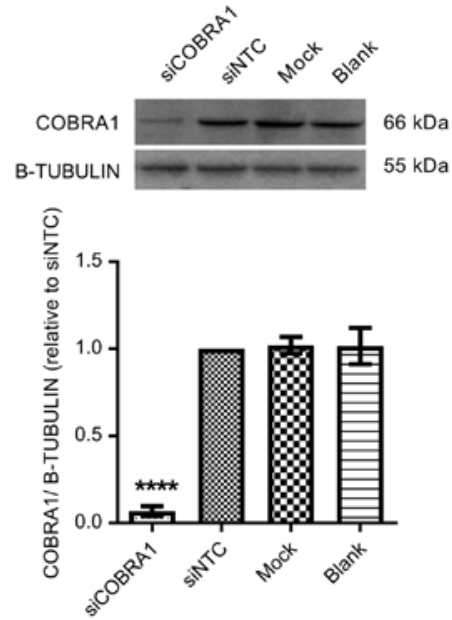

C

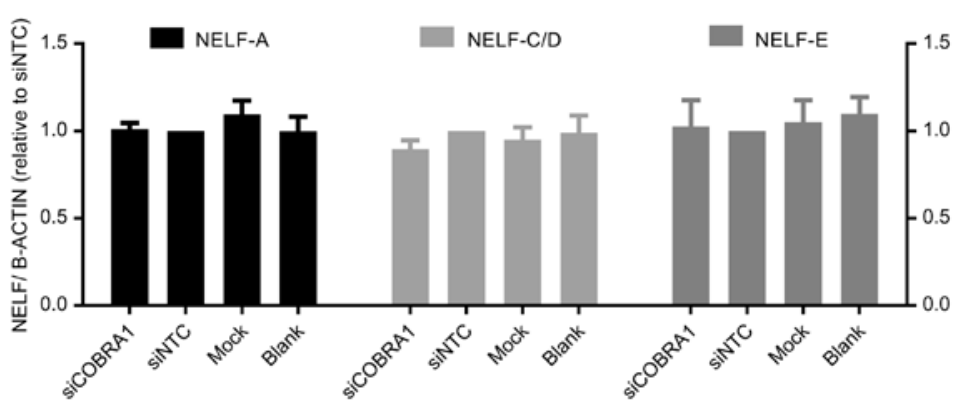

Figure 4. Effect of COBRA1 knockdown on the expression of COBRA1 and the remaining NELF subunits. The efficiency of the pooled COBRA1 siRNA in silencing COBRA1 was assessed at both (A) the RNA and (B) the protein level using RT-PCR and western blotting, respectively. Relative expression is expressed as fold change relative to siNTC. Statistically significant at ${ }^{* * *} \mathrm{p}<0.001,{ }^{* * * *} \mathrm{p}<0.0001$ (one-way ANOVA, Bonferonni's post-test, $\mathrm{n}=3$ ). (C) COBRA1 knockdown does not affect the expression of the remaining NELF subunits. RT-PCR of NELF-A, NELF-C/D and NELF-E transcripts prior and following COBRA1 knockdown. No statistically significant differences were observed ( $\mathrm{p}>0.05$ ) (one-way ANOVA, Bonferonni's post-test, $\mathrm{n}=3$ ). siCOBRA1, COBRA1 siRNA; siNTC, negative siRNA; mock, cells treated with Lipofectamine only; blank, cells left untreated.

cirrhotic or dysplastic tissue for all subunits in the Wurmbach et al dataset (28).

Silencing of COBRA1 does not affect the steady-state transcript levels of the remaining NELF subunits in HepG2. Having noted an upregulated expression pattern for COBRA1 in HCC tumors, we analyzed the effect of silencing COBRA1 on the well-differentiated HCC cell line, HepG2. The HepG2 cell line was among the HCC cell lines that highly express COBRA1 mRNA, as per the Genevestigator database (data not shown). Furthermore, among four different HCC cell lines ranging in grade and tumorigenicity examined recently by our group, the HepG2 cell line exhibited the highest COBRA1 expression at both the RNA and protein levels. It also demonstrated significantly higher COBRA1 expression relative to the normal hepatocyte cell line, MIHA (31). It was hence used here as our cell model. The pooled COBRA1 siRNA was overall successful in silencing COBRA1 expression,resulting on average in $68 \%$ COBRA1 knockdown at the RNA level ( $<<0.001$; Fig. 4A) and a 93\% knockdown at the protein level $(\mathrm{p}<0.0001$; Fig. 4B), relative to the negative siRNA. To investigate whether COBRA1 silencing affected the expression of the remaining NELF subunits, the mRNA expression patterns of NELF-A, NELF-C/D, and NELF-E were compared prior and following COBRA1 knockdown. Interestingly, COBRA1 knockdown did not significantly affect their expression at the RNA level ( $p>0.05$; Fig. 4C).
Silencing of COBRA1 suppresses cell proliferation of Hep $G 2$. To examine the effect of COBRA1 knockdown on cell proliferation, HepG2 cells were transfected with either COBRA1 siRNA or negative siRNA and then harvested and counted at different time-points. Significant suppression was observed in the growth of cells transfected with COBRA1 siRNA at days 3 and 4 post-transfection, relative to control cells (Fig. 5A). Consistent with the cell count data, RT-PCR revealed a significant reduction of $\sim 20 \%$ in the expression of Ki-67 mRNA upon COBRA1 knockdown, when compared to control cells ( $\mathrm{p}<0.01$; Fig. $5 \mathrm{~B}$ ). Ki-67 is a nuclear protein and an important marker of cellular proliferation that is present in all active phases of the cell cycle (G1, S, G2, mitosis) (32).

Silencing of COBRA1 inhibits cellular migration of HepG2. The effect of COBRA1 knockdown on the migratory potential of HepG2 cells was analyzed using a classical scratch wound healing assay. Wound areas analyzed by the TScratch software revealed a $\sim 60 \%$ decrease in the migration rate following COBRA1 knockdown ( $<<0.001$; Fig. 5C). While the control cells showed a $31.86 \%$ wound closure after $24 \mathrm{~h}$, COBRA1 knockdown cells showed a delayed wound closure rate at $13.12 \%$.

Silencing of COBRA1 significantly suppresses survivin gene expression. To explore the underlying mechanisms by which COBRA1 regulates the proliferation and migration of HepG2, 

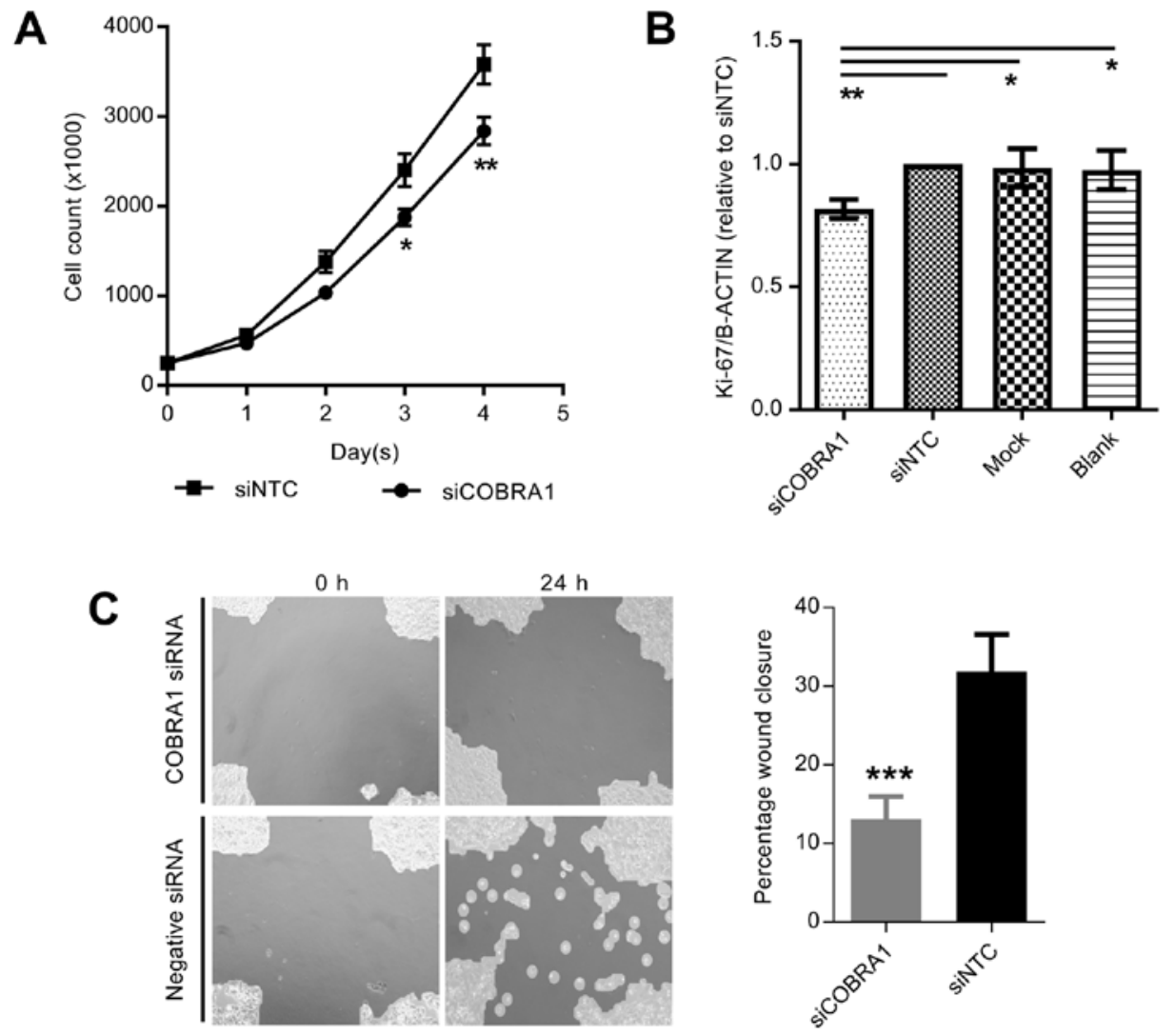

Figure 5. Knockdown of COBRA1 inhibits cellular proliferation and migration of HepG2 cells. (A) The growth rate of HepG2 cells transfected with either COBRA1 siRNA or negative siRNA. Statistically significant at ${ }^{* *} \mathrm{p}<0.01,{ }^{*} \mathrm{p}<0.05$ (two-way ANOVA, Bonferonni's post-test, $\mathrm{n}=2$ ). (B) Semi-quantitative RT-PCR of the cellular proliferation marker, Ki-67. Relative expression is expressed as fold change relative to the siNTC. Statistically significant at ** $<<0.01$, "p $<0.05$ (one-way ANOVA, Bonferonni's post-test, $n=3$ ). (C) Migration of HepG2 cells prior and following knockdown of COBRA1 in a scratch wound healing assay. Wound areas determined using TScratch software were used to calculate percentage wound closure rates. Statistically significant at ${ }^{* * * *} \mathrm{p}<0.001$ (Student's t-test, two-tailed, $n=3$ ). siCOBRA1, COBRA1 siRNA; siNTC, negative siRNA.

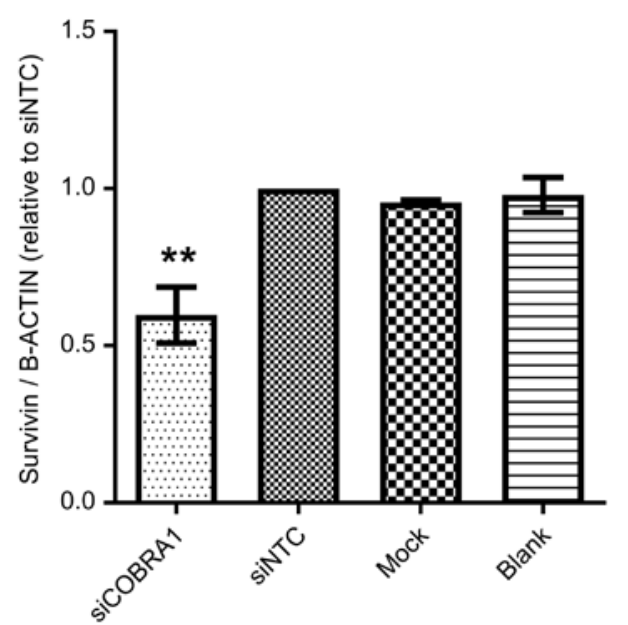

Figure 6. Silencing of COBRA1 suppresses survivin (BIRC5) expression. Semi-quantitative RT-PCR of survivin at $72 \mathrm{~h}$ post-transfection. Normalized expression is expressed as fold change relative to the siNTC. Statistically significant at ${ }^{* *} \mathrm{p}<0.01$ (one-way ANOVA, Bonferonni's post-test, $\mathrm{n}=3$ ). siCOBRA1, COBRA1 siRNA; siNTC, negative siRNA.

the expression of genes commonly reported deregulated in cancer such as survivin were examined. Of the three transcript variants of survivin analyzed, wild-type (WT) survivin, survivin-2B, and survivin-deltaex3, the WT survivin transcript represented the dominant survivin variant in HepG2.
Silencing of COBRA1 significantly suppressed the expression of WT survivin by $40 \%$ compared with cells transfected with control siRNA (p<0.01; Fig. 6).

\section{Discussion}

Despite the different treatment regimens available, the prognosis of HCC remains very poor with an overall 5-year survival rate of $<5 \%$ (33), making it the second most common cause of cancer-related mortality worldwide (2). In this study, our interest was in one of the genes recently implicated in the progression of different cancers, known as COBRA1 $(17,18)$. Here, we propose a potential role for COBRA1 in HCC tumorigenesis and provide evidence highlighting its significance in supporting HCC proliferation and migration.

In this study, COBRA1 overexpression was observed in HCC tumor tissues when compared to their adjacent non-tumor counterparts. COBRA1 overexpression was further confirmed in three different datasets retrieved from the Oncomine database. Collectively, data presented here demonstrates that the overexpression of COBRA1 is a frequent event in the hepatocytes' normal-to-tumor transition. While samples from the Wurmbach et al study were from patients with an HCV etiology, the large majority of those in the Roessler et al (27) study were from an HBV background, strongly suggesting that the overexpression of COBRA1 is a feature associated with both etiologies of HCC. 
Given COBRA1's role in transcriptional regulation, a deregulation in the expression of COBRA1 might result in a similar deregulation in all of its downstream genes and hence, our hypothesis for a role for COBRA1 in HCC. To test this hypothesis, RNA interference was used to silence COBRA1 expression. Here, our data indicates that COBRA1 is essential for maintaining the cellular proliferation rates of the HepG2 cell line as shown by both the reduced cell count and the decrease in $\mathrm{Ki}-67$ expression upon COBRA1 knockdown. Even though little is known about the function of Ki-67, its presence is believed to be an absolute requirement for cell cycle progression and the maintenance of cellular proliferation (34). This positive effect that COBRA1 has in mediating the growth of HCC goes in line with its role in maintaining the transcription of genes involved in cell cycle control, cell proliferation, cell death and DNA repair $(15,16)$. This is not the first time COBRA1 has been implicated in the proliferation of cancer. However, in contrast, Aiyar et al demonstrated the importance of COBRA1 in suppressing the estrogen-dependent growth of breast cancer cells (9).

Local invasion and distant metastasis are other key hallmarks of cancer and indicators of poor prognosis (35). In line with COBRA1's established role in breast cancer as a tumor suppressor, lack of COBRA1 expression is associated with distant metastasis and recurrence in breast cancer patients and thus considered an indicator of poor prognosis (17). In agreement, the expression pattern of many previously reported COBRA1-regulated genes have been associated with advanced and/or metastatic breast cancer $(9,15,16)$. In contrast to breast cancer where COBRA1's expression is inversely correlated with metastasis, data findings from our in vitro study suggest an active role for COBRA1 in cell migration; however whether that leads to enhanced invasion is yet to be examined. The molecular basis behind this apparent tissue-specific nature of COBRA1s' functions remains unknown and warrants further study.

Due to the small sample size of our study, no attempt was made here to correlate COBRA1 expression to different clinicopathological parameters statistically and therefore, the prognostic value of COBRA1 expression in HCC remains to be determined. It is thus unclear whether the high COBRA1 expression is associated with distant metastasis and tumor recurrence in patients. Nevertheless, it is important to mention that the pattern of COBRA1 overexpression was not consistent in all HCC tissue samples or cell lines examined here or reported previously by our group (31) that were clinically associated with high grade, advanced stage and/or invasive traits. This demonstrates the heterogeneity of HCC and underlying complexity of COBRA1's role in HCC, which requires further study and a more comprehensive analysis.

The deregulation of cell proliferation and migration in cancer is usually associated with the deregulation of multiple mechanisms that normally exist to suppress tumor formation and metastasis. Here, data findings from our study show that survivin might be one of the mechanisms by which COBRA1 mediates its role on the growth and migration of HCC. Survivin is an oncogene that is commonly upregulated in almost all human malignancies including HCC and is known to play key roles in cellular proliferation and survival (36). It is a member of the IAP family of proteins whose expression correlates with a more aggressive disease and a poor prognosis (37). Besides the 4-exon WT survivin transcript, at least six alternatively spliced variants have been identified to date (38). Of those, only three have been extensively studied and were shown to represent nearly $98 \%$ of the mRNA expression of the survivin gene. These are the WT survivin, survivin-2B, and survivin- $\Delta \mathrm{Ex} 3$ (39). Our results show that of these three transcripts which were examined in our study, WT survivin represents the dominant splice variant expressed in HCC which goes in line with previous literature (40). In HCC, high levels of WT survivin mRNA correlate with a more advanced cancer. While the mRNA expression levels of WT survivin and survivin- $\Delta \mathrm{Ex} 3$ correlate with high proliferative activity, that of survivin-2B does not (41). The suppression of survivin expression upon COBRA1 knockdown suggests that survivin might be one of the mechanisms by which COBRA1 mediates its involvement in HCC growth and migration. Further experiments are still required to determine whether COBRA1 is a direct regulator of survivin expression.

NELF is a 4-subunit complex that is activated with the assembly of its four subunits; NELF-A, NELF-B (or COBRA1), NELF-C/D and NELF-E. NELF-C/D together with COBRA1 form the core of the NELF complex, bridging the NELF-A and NELF-E subunits which contain the RNAPII and RNA binding domains, respectively. Even though the NELF-A and NELF-E are the binding domains by which the NELF complex will bind to RNAPII and RNA respectively, all four subunits are necessary for the assembly and function of the complex (7). In fact, this explains the well-established interdependent nature of the NELF subunits, whereby knockdown of any NELF subunit results in the simultaneous co-depletion of the remaining subunits $(8,17,30)$. Here, knockdown of COBRA1 had no effect on the expression of the remaining subunits at the RNA level. This goes in line with previous literature whereby the reported simultaneous co-depletion of the remaining NELF subunits occurred exclusively at a posttranscriptional level $(8,17,30)$. Therefore, further examination is still required before we can draw a conclusion here.

Given this interdependent nature of the NELF subunits, a disease-based deregulation in the expression pattern of one subunit is expected to reflect equally in the remaining subunits. This was observed in our in silico data, whereby the expression pattern of the NELF subunits (NELF-A, NELD-C/D and NELF-E) in HCC tumors mirrored that of COBRA1 when compared to non-tumor or normal liver tissue. Hence, it is tempting to speculate similar physiological functions for the different NELF subunits as well as roles in pathological conditions. Limited with the small number of studies, this pattern has been observed so far in breast cancer. Both COBRA1 and NELF-C/D were reported independently to have negative roles in the growth and progression of breast cancer. In addition, the expression of either negatively correlated with the aggressiveness of breast cancer $(17,42)$. Here, our results follow the same pattern and go in line with previous data published regarding other NELF subunits in HCC. NELF-E (or RDBP) was reported an independent risk factor for intrahepatic recurrence, with a higher expression pattern in HCC tumors versus adjacent non-tumor tissues. Furthermore, NELF-E silencing resulted in a decrease in the proliferation of the hepatoma HLE cell line, with no significant changes observed in cell cycle 
distribution (43). In fact, the dedifferentiation process of HCC, one of the main events that define the multi-step progression of hepatocarcinogenesis, was shown associated with the overexpression of NELF-E among others (19).

In conclusion, COBRA1's role in cancer seems to be highly cancer type-dependent. While previously reported a tumor suppressor in breast cancer with an expression pattern that directly correlated with prognosis, data in upper gastrointestinal carcinomas seem to support its role as an oncogene $(17,18)$. In the field of cancer, the role of COBRA1 is not yet fully understood, and much of the data that currently exists is conflicting. Nonetheless, data that exist demonstrate an important role for COBRA1 in cancer and the fact that much of it is conflicting highlights the underlying complexity of the matter. Here in HCC, our data findings collectively highlight an important role for COBRA1 in supporting HCC proliferation and migration; whether this role, however, is similar to that previously reported in UGC remains to be determined.

\section{Acknowledgements}

This study was supported by the American University in Cairo Internal Faculty Research Grant to Asma Amleh and Graduate Student Research Grants to Eman El Zeneini and Sarah Kamel. We would like to thank Professor Mohamed Baha, Hany Dabboss and Rasha Refaiy for aiding in the collection of the clinical HCC specimens used in the study.

\section{References}

1. Jemal A, Bray F, Center MM, Ferlay J, Ward E and Forman D: Global cancer statistics. CA Cancer J 61: 69-90, 2011.

2. Ferlay J, Soerjomataram I, Ervik M, Dikshit R, Eser S, Mathers C, Rebelo M, Parkin DM, Forman D and Bray F: GLOBOCAN 2012 v1.0, Cancer Incidence and Mortality Worldwide: IARC CancerBase No. 11 (Internet). International Agency for Research on Cancer, Lyon, France, 2013. http://globocan.iarc.fr. Accessed July 22, 2015.

3. Capece D, Fischietti M, Verzella D, Gaggiano A, Cicciarelli G, Tessitore A, Zazzeroni F and Edoardo Alesse E: The inflammatory microenvironment in hepatocellular carcinoma: A pivotal role for tumor-associated macrophages. Biomed Res Int 2013 : 187204, 2013.

4. Farazi PA and DePinho RA: Hepatocellular carcinoma pathogenesis: From genes to environment. Nat Rev Cancer 6: 674-687, 2006.

5. Cornellà $\mathrm{H}$, Alsinet $\mathrm{C}$ and Villanueva A: Molecular pathogenesis of hepatocellular carcinoma. Alcohol Clin Exp Res 35: 821-825, 2011.

6. Ye Q, Hu YF, Zhong H, Nye AC, Belmont AS and Li R: BRCA1induced large-scale chromatin unfolding and allele-specific effects of cancer-predisposing mutations. J Cell Biol 155: 911-921, 2001

7. Narita T, Yamaguchi Y, Yano K, Sugimoto S, Chanarat S, Wada T, Kim DK, Hasegawa J, Omori M, Inukai N, et al: Human transcription elongation factor NELF: Identification of novel subunits and reconstitution of the functionally active complex. Mol Cell Biol 23: 1863-1873, 2003.

8. Sun J and Li R: Human negative elongation factor activates transcription and regulates alternative transcription initiation. J Biol Chem 285: 6443-6452, 2010

9. Aiyar SE, Sun JL, Blair AL, Moskaluk CA, Lu YZ, Ye QN, Yamaguchi Y, Mukherjee A, Ren DM, Handa H, et al: Attenuation of estrogen receptor $\alpha$-mediated transcription through estrogenstimulated recruitment of a negative elongation factor. Genes Dev 18: 2134-2146, 2004

10. Persson I: Estrogens in the causation of breast, endometrial and ovarian cancers - evidence and hypotheses from epidemiological findings. J Steroid Biochem Mol Biol 74: 357-364, 2000.
11. Foster JS, Henley DC, Ahamed S and Wimalasena J: Estrogens and cell-cycle regulation in breast cancer. Trends Endocrinol Metab 12: 320-327, 2001.

12. Sun J, Blair AL, Aiyar SE and Li R: Cofactor of BRCA1 modulates androgen-dependent transcription and alternative splicing. J Steroid Biochem Mol Biol 107: 131-139, 2007.

13. Lonergan PE and Tindall DJ: Androgen receptor signaling in prostate cancer development and progression. J Carcinog 10: 20 , 2011.

14. Ma W-L, Lai H-C, Yeh S, Cai X and Chang C: Androgen receptor roles in hepatocellular carcinoma, fatty liver, cirrhosis and hepatitis. Endocr Relat Cancer 21: R165-R182, 2014.

15. Aiyar SE, Cho H, Lee J and Li R: Concerted transcriptional regulation by BRCA1 and COBRA1 in breast cancer cells. Int J Biol Sci 3: 486-492, 2007.

16. Aiyar SE, Blair AL, Hopkinson DA, Bekiranov S and Li R: Regulation of clustered gene expression by cofactor of BRCA1 (COBRA1) in breast cancer cells. Oncogene 26: 2543-2553, 2007.

17. Sun J, Watkins G, Blair AL, Moskaluk C, Ghosh S, Jiang WG and Li R: Deregulation of cofactor of BRCA1 expression in breast cancer cells. J Cell Biochem 103: 1798-1807, 2008.

18. McChesney PA, Aiyar SE, Lee OJ, Zaika A, Moskaluk C, Li R and El-Rifai W: Cofactor of BRCA1: A novel transcription factor regulator in upper gastrointestinal adenocarcinomas. Cancer Res 66: 1346-1353, 2006.

19. Midorikawa Y, Tsutsumi S, Taniguchi H, Ishii M, Kobune Y, Kodama T, Makuuchi M and Aburatani H: Identification of genes associated with dedifferentiation of hepatocellular carcinoma with expression profiling analysis. Jpn J Cancer Res 93: 636-643, 2002.

20. Zheng J-N, Ma T-X, Cao J-Y, Sun X-Q, Chen J-C, Li W, Wen RM, Sun YF and Pei DS: Knockdown of Ki-67 by small interfering RNA leads to inhibition of proliferation and induction of apoptosis in human renal carcinoma cells. Life Sci 78: 724-729, 2006.

21. Chen WC, Liu Q, Fu JX and Kang SY: Expression of survivin and its significance in colorectal cancer. World J Gastroenterol 10: 2886-2889, 2004.

22. Liang C-CC-C, Park AY and Guan J-LJ-L: In vitro scratch assay: A convenient and inexpensive method for analysis of cell migration in vitro. Nat Protoc 2: 329-333, 2007.

23. Gebäck T, Schulz MMP, Koumoutsakos P and Detmar M: TScratch: A novel and simple software tool for automated analysis of monolayer wound healing assays. Biotechniques 46: 265-274, 2009.

24. Rhodes DR, Yu J, Shanker K, Deshpande N, Varambally R, Ghosh D, Barrette T, Pandey A and Chinnaiyan AM: ONCOMINE: A cancer microarray database and integrated data-mining platform. Neoplasia 6: 1-6, 2004.

25. Chen X, Cheung ST, So S, Fan ST, Barry C, Higgins J, Lai KM, Ji J, Dudoit S, Ng IO, et al: Gene expression patterns in human liver cancers. Mol Biol Cell 13: 1929-1939, 2002.

26. Mas VR, Maluf DG, Archer KJ, Yanek K, Kong X, Kulik L, Freise CE, Olthoff KM, Ghobrial RM, et al: Genes involved in viral carcinogenesis and tumor initiation in hepatitis $\mathrm{C}$ virusinduced hepatocellular carcinoma. Mol Med 15: 85-94, 2009.

27. Roessler S, Jia H-L, Budhu A, Forgues M, Ye Q-H, Lee J-S, Thorgeirsson SS, Sun Z, Tang Z-Y, Qin L-X, et al: A unique metastasis gene signature enables prediction of tumor relapse in early-stage hepatocellular carcinoma patients. Cancer Res 70: 10202-10212, 2010.

28. Wurmbach E, Chen YB, Khitrov G, Zhang W, Roayaie S, Schwartz M, Fiel I, Thung S, Mazzaferro V, Bruix J, et al: Genome-wide molecular profiles of HCV-induced dysplasia and hepatocellular carcinoma. Hepatology 45: 938-947, 2007.

29. Hruz T, Laule O, Szabo G, Wessendorp F, Bleuler S, Oertle L, Widmayer P, Gruissem W and Zimmermann P: Genevestigator V3: A reference expression database for the meta-analysis of transcriptomes. Adv Bioinformatics 2008: 420747, 2008.

30. Narita T, Yung TMC, Yamamoto J, Tsuboi Y, Tanabe H, Tanaka K, Yamaguchi Y and Handa H: NELF interacts with CBC and participates in 3 ' end processing of replication-dependent histone mRNAs. Mol Cell 26: 349-365, 2007.

31. Youssef A, Shawer H, Afify A and Amleh A: The potential involvement of the cofactor of BRCA1 in hepatocellular carcinoma pathogenesis. Adv Mod Oncol Res: Aug 10, 2016 (Epub ahead of print). doi: 10.18282/amor.v2.i4.129.

32. Scholzen T and Gerdes J: The Ki-67 protein: From the known and the unknown. J Cell Physiol 182: 311-322, 2000. 
33. Huang X, Zheng Q and Yuan Z: Neoadjuvant chemotherapy for hepatocellular carcinoma. In: Hepatocellular Carcinoma - Future Outlook. Kaseb A (ed). http://www.intechopen.com/ books/hepatocellular-carcinoma-future-outlook. Accessed July 22, 2015.

34. Schlüter C, Duchrow M, Wohlenberg C, Becker MH, Key G, Flad HD and Gerdes J: The cell proliferation-associated antigen of antibody Ki-67: A very large, ubiquitous nuclear protein with numerous repeated elements, representing a new kind of cell cycle-maintaining proteins. J Cell Biol 123: 513-522, 1993.

35. Hanahan D and Weinberg RA: Hallmarks of cancer: The next generation. Cell 144: 646-674, 2011.

36. Fukuda S and Pelus LM: Survivin, a cancer target with an emerging role in normal adult tissues. Mol Cancer Ther 5: 1087-1098, 2006.

37. Jaiswal PK, Goel A and Mittal RD: Survivin: A molecular biomarker in cancer. Indian J Med Res 141: 389-397, 2015.

38. Pavlidou A, Kroupis C and Dimas K: Association of survivin splice variants with prognosis and treatment of breast cancer. World J Clin Oncol 5: 883-894, 2014.

39. Mull AN, Klar A and Navara CS: Differential localization and high expression of SURVIVIN splice variants in human embryonic stem cells but not in differentiated cells implicate a role for SURVIVIN in pluripotency. Stem Cell Res 12: 539-549, 2014.
40. Kannangai R, Wang J, Liu QZ, Sahin F and Torbenson M: Survivin overexpression in hepatocellular carcinoma is associated with p53 dysregulation. Int J Gastrointest Cancer 35: 53-60, 2005.

41. Takashima H, Nakajima T, Moriguchi M, Sekoguchi S, Nishikawa T, Watanabe T, Katagishi T, Kimura H, Minami M, Itoh Y, et al: In vivo expression patterns of survivin and its splicing variants in chronic liver disease and hepatocellular carcinoma. Liver Int 25: 77-84, 2005.

42. Zou W, Yang Y, Wu Y, Sun L, Chi Y, Wu W, Yun X, Xie J and $\mathrm{Gu} \mathrm{J}$ : Negative role of trihydrophobin 1 in breast cancer growth and migration. Cancer Sci 101: 2156-2162, 2010.

43. Iida M, Iizuka N, Tsunedomi R, Tsutsui M, Yoshida S, Maeda Y, Tokuhisa Y, Sakamoto K, Yoshimura K, Tamesa T, et al: Overexpression of the RD RNA binding protein in hepatitis C virus-related hepatocellular carcinoma. Oncol Rep 28: 728-734, 2012. 